\title{
Automatically Diagnosing Disk Bulge and Disk Herniation With Lumbar Magnetic Resonance Images by Using Deep Convolutional Neural Networks: Method Development Study
}

Qiong Pan ${ }^{1,2^{*}}$, MS; Kai Zhang ${ }^{3,4^{*}}, \mathrm{PhD}$; Lin He ${ }^{3}$, BSc; Zhou Dong ${ }^{5}$, MS; Lei Zhang ${ }^{3}$, BSc; Xiaohang Wu ${ }^{6}, \mathrm{PhD}$ Yi $\mathrm{Wu}^{7}, \mathrm{MD}$; Yanjun $\mathrm{Gao}^{8}, \mathrm{PhD}$

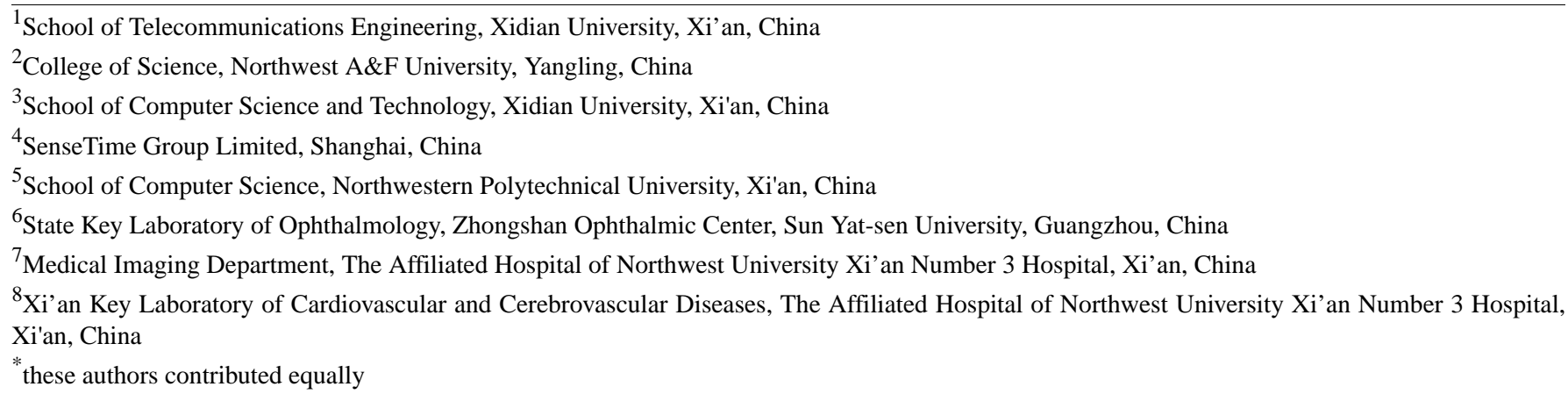

Corresponding Author:

Yanjun Gao, PhD

Xi' an Key Laboratory of Cardiovascular and Cerebrovascular Diseases

The Affiliated Hospital of Northwest University Xi' an Number 3 Hospital

No 10 Eastern section of the third FengCheng Rd

WeiYang District

Xi'an

China

Phone: 8661816169

Fax: 8661816100

Email: nige.001@stu.xjtu.edu.cn

\section{Abstract}

Background: Disk herniation and disk bulge are two common disorders of lumbar intervertebral disks (IVDs) that often result in numbness, pain in the lower limbs, and lower back pain. Magnetic resonance (MR) imaging is one of the most efficient techniques for detecting lumbar diseases and is widely used for making clinical diagnoses at hospitals. However, there is a lack of efficient tools for effectively interpreting massive amounts of MR images to meet the requirements of many radiologists.

Objective: The aim of this study was to present an automatic system for diagnosing disk bulge and herniation that saves time and can effectively and significantly reduce the workload of radiologists.

Methods: The diagnosis of lumbar vertebral disorders is highly dependent on medical images. Therefore, we chose the two most common diseases - disk bulge and herniation—as research subjects. This study is mainly about identifying the position of IVDs (lumbar vertebra [L] 1 to L2, L2-L3, L3-L4, L4-L5, and L5 to sacral vertebra [S] 1) by analyzing the geometrical relationship between sagittal and axial images and classifying axial lumbar disk MR images via deep convolutional neural networks.

Results: This system involved 4 steps. In the first step, it automatically located vertebral bodies (including the L1, L2, L3, L4, L5, and S1) in sagittal images by using the faster region-based convolutional neural network, and our fourfold cross-validation showed $100 \%$ accuracy. In the second step, it spontaneously identified the corresponding disk in each axial lumbar disk MR image with $100 \%$ accuracy. In the third step, the accuracy for automatically locating the intervertebral disk region of interest in axial MR images was 100\%. In the fourth step, the 3-class classification (normal disk, disk bulge, and disk herniation) accuracies for the L1-L2, L2-L3, L3-L4, L4-L5, and L5-S1 IVDs were 92.7\%, 84.4\%, 92.1\%, 90.4\%, and 84.2\%, respectively.

Conclusions: The automatic diagnosis system was successfully built, and it could classify images of normal disks, disk bulge, and disk herniation. This system provided a web-based test for interpreting lumbar disk MR images that could significantly 
improve diagnostic efficiency and standardized diagnosis reports. This system can also be used to detect other lumbar abnormalities and cervical spondylosis.

(JMIR Med Inform 2021;9(5):e14755) doi: 10.2196/14755

\section{KEYWORDS}

deep learning; object localization; disk herniation; disk bulge; image classification

\section{Introduction}

Magnetic resonance imaging (MRI) is a widely used technique for detecting lumbar disorders, and its advantages include high image quality and noninvasive and ionization-free radiation. Disk herniation and disk bulge are two common types of lumbar intervertebral disk (IVD) injuries that often result in low back pain and tingling and numbness in the legs $[1,2]$. The diagnosis of disk disorders is highly dependent on radiology methods such as MRI. The leading question is as follows: how can radiologists interpret massive amounts of magnetic resonance (MR) images quickly and accurately for real-world applications? Motivated by machine learning-and deep learning-based clinical practice [3-6], we propose an automatic diagnosis system for diagnosing disk bulge and disk herniation with MR images via deep convolutional neural networks (CNNs), which can reduce radiologists' workload and provide the consistency required to produce standardized diagnosis reports.

Koh et al [7] proposed a computer-aided framework that uses several heterogeneous classifiers (ie, a perceptron classifier, a least mean squares classifier, a support vector machine classifier, and a k-means classifier) to construct a 2-level classification scheme for disk herniation diagnosis, which achieved $99 \%$ accuracy for 70 subjects. A probability classifier based on Gaussian models was proposed to detect abnormal IVDs. This model used the following three features: appearance, location, and context [8]. A study [9] on texture features that were obtained from IVD MR images used three different classifiers (ie, the back-propagation neural network, k-nearest neighbor, and support vector machine classifiers) to classify normal disks and IVDs and achieved a maximum accuracy of $83.33 \%$. Additionally, many other methods have been proposed to automatically diagnose IVD diseases based on MR images [10-13]. Most of these models are for sagittal MR images, and there are very few studies that have used axial lumbar MR images, which are even more important in real clinical scenarios to identify disk bulge and herniation [13]. Most previous studies have mainly focused on binary classification (disease and normal) $[7-9,11,12]$, as it is rare to study 2 diseases at the same time. In this study, we present a deep CNN-based diagnosis system for diagnosing lumbar disk bulge and disk herniation based on axial MR images. CNN analysis has proven to be an efficient method that is widely used to solve various image problems and has achieved huge success in many applicable fields [14-18].

This study aimed to develop a clinical applicable system that requires as little information from doctors as possible for diagnosing disk bulge and disk herniation via deep learning methods [19-21].

\section{Methods}

\section{Data Set}

In this study, lumbar MR Images and clinical diagnosis reports were collected from the Medical Imaging Department of Xi' an Number 3 Hospital, which is a large-scale grade $3 \mathrm{~A}$ general hospital in Xi'an, China. The sagittal and axial $\mathrm{T}_{2}$-weighted lumbar MR images of 500 patients were acquired by using a Philips Ingenia 3.0T scanner and exported in the Digital Imaging and Communications in Medicine (DICOM) format. The main diagnosis was based on axial images, as they display the morphology of IVDs more clearly than other images. For each subject, midsagittal images were used to locate IVDs in axial images. A total of 3555 axial images were used in this study. These images were labeled as normal disk, disk bulge, and disk herniation according to diagnosis reports and rechecked by an experienced radiologist, as shown in Table 1. Examples of midsagittal lumbar images and axial images of normal disks, disk bulge, and disk herniation are shown in Figure 1.

Table 1. The number of axial images in each category.

\begin{tabular}{|c|c|c|c|c|}
\hline Intervertebral disk & Normal images, $\mathrm{n}$ & Bulge images, $\mathrm{n}$ & Herniation images, $\mathrm{n}$ & Total, $\mathrm{n}$ \\
\hline L1-L2 ${ }^{\mathrm{a}}$ & 593 & 37 & 36 & 666 \\
\hline L2-L3 & 549 & 120 & 30 & 699 \\
\hline L3-L4 & 347 & 284 & 86 & 717 \\
\hline L4-L5 & 158 & 413 & 178 & 749 \\
\hline $\mathrm{L} 5-\mathrm{S}^{\mathrm{b}}$ & 238 & 242 & 244 & 724 \\
\hline All intervertebral disks & 1885 & 1096 & 574 & 3555 \\
\hline
\end{tabular}

${ }^{\mathrm{a}} \mathrm{L}$ : lumber vertebra.

${ }^{\mathrm{b}} \mathrm{S}$ : sacral vertebra. 
Figure 1. Examples of lumbar MR images. (A) A sagittal lumbar MR image in which 5 IVDs are labeled. (B) A sagittal lumbar MR image in which 6 vertebral bodies are enclosed in boxes. (C) An axial lumbar MR image of a normal disk. (D) An axial lumbar MR image of disk bulge. (E) An axial lumbar MR image of disk herniation. L: lumbar vertebra; MR: magnetic resonance; S: sacral.

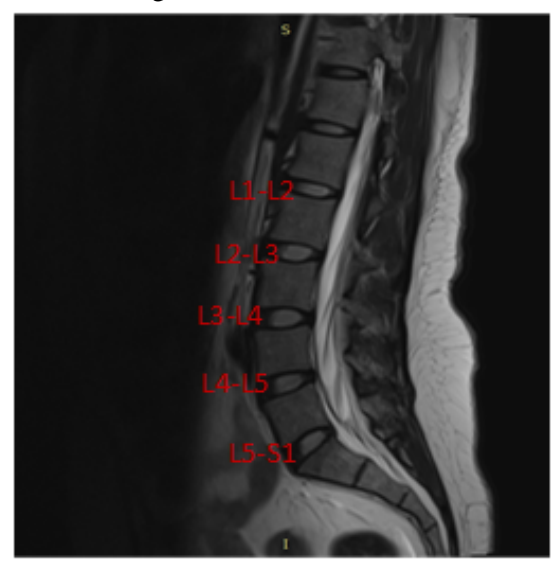

A

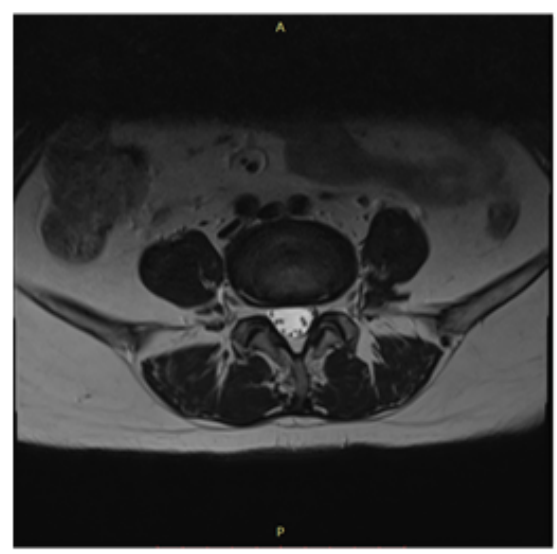

D

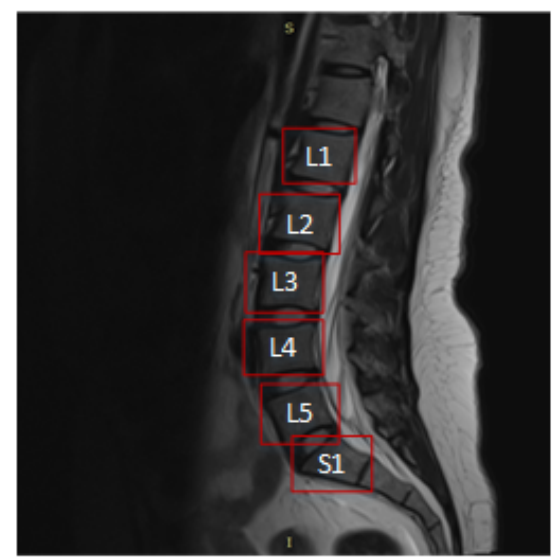

B

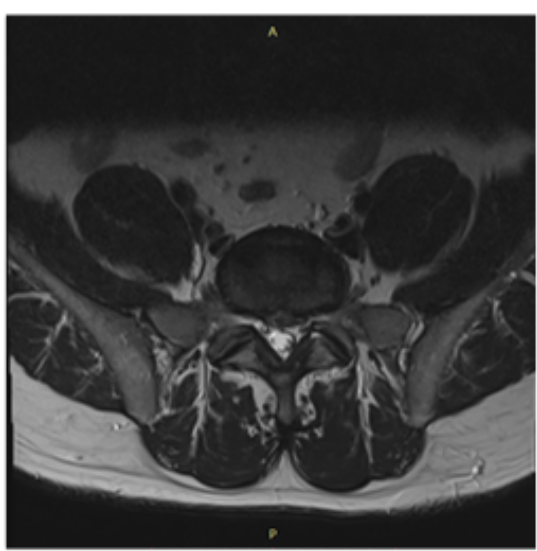

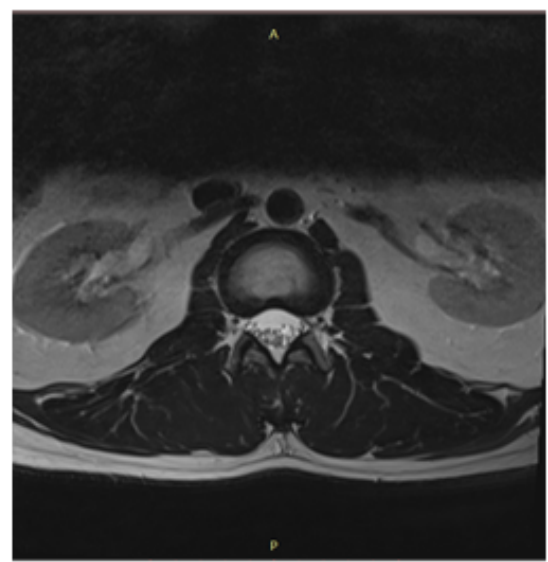

C

\section{Overall Diagnosis System}

Our system consists of 4 steps, as shown in Figure 2. In the first step, the six lumbar vertebral bodies (lumbar vertebra [L] 1, L2, L3, L4, L5, and sacral vertebra [S] 1) in midsagittal images were detected and located. The second step was to identify the corresponding IVDs in each axial MR image. Afterward, these axial images were grouped into five categories (L1-L2, L2-L3, L3-L4, L4-L5, and L5-S1). In the third step, the IVD regions of interest (ROIs) in axial images were segmented to decrease the noise of the images. In the fourth step, each ROI image that included the five IVDs was classified as normal disk, disk bulge, or disk herniation. 
Figure 2. Overall diagnosis system. This system consists of 4 steps. First, vertebral bodies (L1, L2, L3, L4, and L5) in sagittal lumbar magnetic resonance images were automatically located by using the faster R-CNN, and the middle point of each vertebral body was calculated. Second, the axial images were grouped into 5 categories. Each category corresponded to an intervertebral disk (ie, the L1-L2, L2-L3, L3-L4, L4-L5, and L5-S1 intervertebral disks). Third, the intervertebral disk regions of interest in each axial MR image were segmented using the faster R-CNN. Finally, in each category, the region-of-interest images were classified as images of normal disks, disk bulge, and disk herniation using ResNet101. L: lumbar vertebra; R-CNN: region-based convolutional neural network; S: sacral.

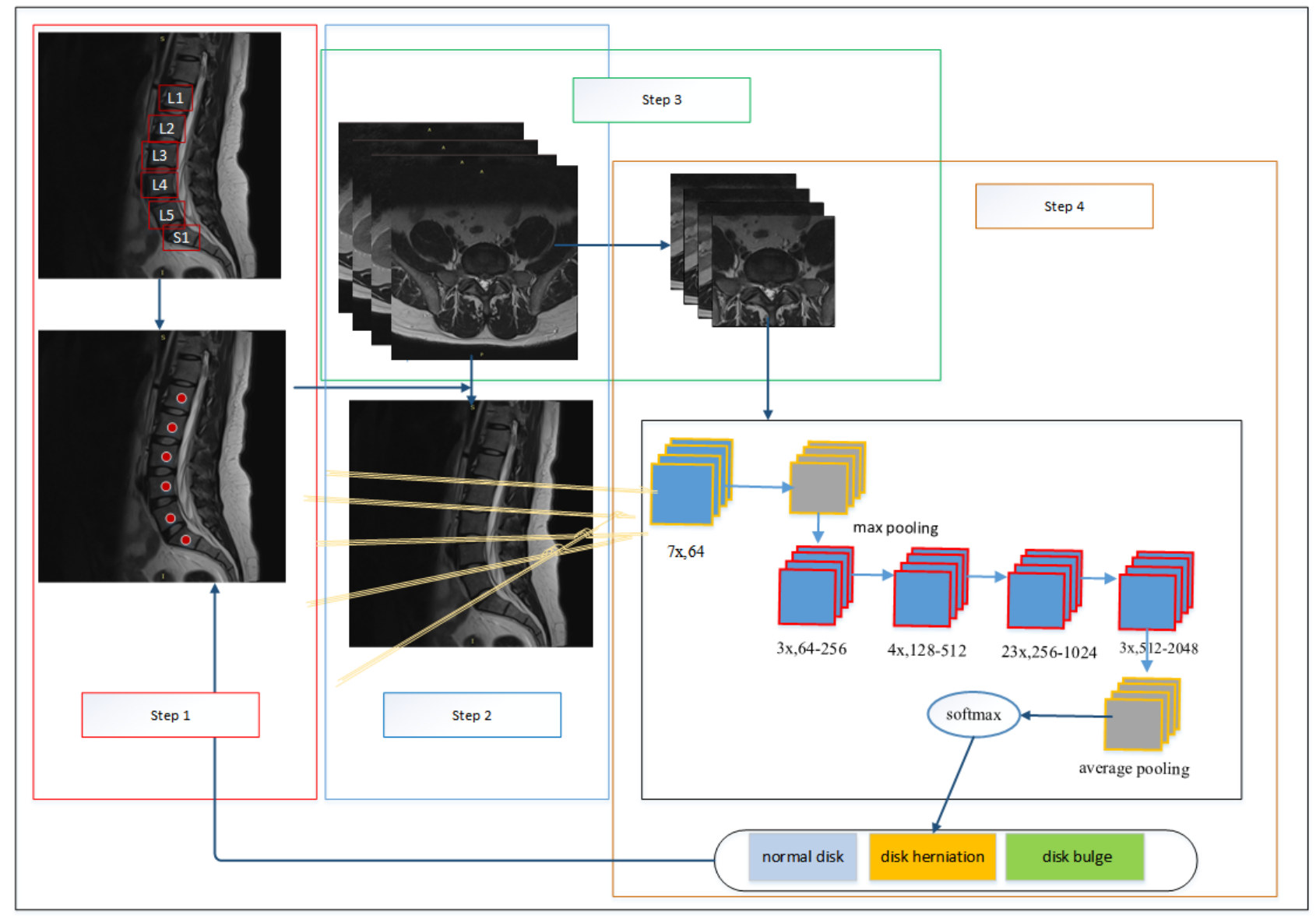

\section{Automatically Locating Vertebral Bodies in Midsagittal Images}

The faster region-based CNN (R-CNN) [19] was developed from the R-CNN [22] and the fast R-CNN [23], which unifies the target detection process (including candidate region generation, feature extraction, classification, and position refinement) into 1 deep network framework and greatly improves operational speed. In step 1, the faster R-CNN was used to locate the vertebral bodies in sagittal MR images.

First, the six vertebral bodies (L1-S1) in 200 midsagittal images were manually located under the guidance of a radiologist. Second, the faster R-CNN was trained to detect and locate each vertebral body. We detected vertebral bodies instead of disks because they were easier to manually locate. Finally, the middle point coordinate of each vertebral body was calculated based on bounding box coordinates, as the precise location of the vertebral bodies would be used to locate the vertebrae in axial MR images, as shown in Figure 1 (step 1).

The faster R-CNN was implemented with Caffe [24] (Berkeley Vision and Learning Center deep learning framework) and trained in parallel on 4 Nvidia Titan $\mathrm{X}$ graphics processing units.
Accuracy, sensitivity, and specificity $[25,26]$ were analyzed to comprehensively evaluate the performance of this system.

\section{Identifying the Corresponding IVD in Each Axial MR Image}

For each subject, 15 axial slices were needed to identify the corresponding IVDs (L1-L2, L2-L3, L3-L4, L4-L5, and L5-S1) in each axial MR image. In step 1, the center point coordinates of the six vertebral bodies in the sagittal images were calculated. The directed distances from these center points to each axial image were calculated for each subject based on the spatial location relationship between sagittal images and axial images. The directed distances indicated which IVDs were closer to the corresponding IVDs in each axial image and which IVDs were located above or below the corresponding IVDs, as shown in Figure 3. Based on these distances, the axial slices were classified into 5 categories (L1-L2, L2-L3, L3-L4, L4-L5, and L5-S1). The conversion from DICOM patient-based coordinates to $2 \mathrm{D}$ computer coordinates was conducted in order to establish the relationship between the primitively processed images and the 3D DICOM coordinates. The detailed procedures are depicted in Multimedia Appendix 1. 
Figure 3. The intervertebral disks (from L1-L2 to L5-S1) in each axial image were located by calculating directed distances. The red dot shows the middle point of each vertebral body in a sagittal image. The blue line depicts the directed distance from the red dot to a specific axial image. L: lumbar vertebra; S: sacral.

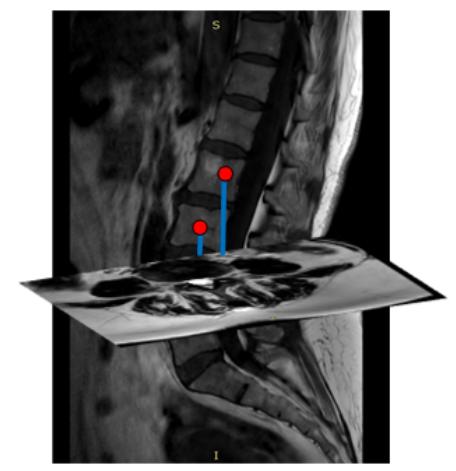

\section{Locating IVD ROIs in Axial MR Images}

Axial lumbar MR images contain large amounts of unrelated areas. In order to focus on IVDs and extract more relevant features, IVD areas were labeled manually in 1237 axial images, including normal disk areas, bulging disk areas, disk herniation areas, and the L1-L2 to L5-S1 IVD areas. The IVD areas of each ROI image needed to be located to train the faster R-CNN, and our fourfold cross-validation showed $100 \%$ accuracy. Afterward, the ROIs in each axial lumbar image were detected and extracted using the faster R-CNN, as shown in Figure 2 (step 3). We reserved a larger area for the components surrounding IVDs, as they may also help with identifying the condition of the disks (eg, the compression of the spinal canal).

\section{Classification of ROI Images}

It is worth mentioning that the degradation problem of the ultradeep CNN may result in reduced classification accuracy as the depth of the CNN increases. He et al [27] proposed a deep residual network framework that can solve this problem by using the residual block method, and this was proven to have significant accuracy for the ImageNet validation set [27-29]. The residual architecture of ResNet101 is shown in Figure 2 (step 4).

According to the diagnosis reports, in every category (L1-L2 to L5-S1), a total of 3555 axial MR images were labeled as normal disk, disk bulge, or disk herniation. All 3555 ROI images were reviewed by an expert radiologist to confirm whether the images conformed to the labels. Afterward, ResNet101 was used to conduct the 3-class classification for each category, and our fourfold cross-validation showed classification accuracies of $92.7 \%, 84.4 \%, 92.1 \%, 90.4 \%$ and $84.2 \%$ for the L1-L2, L2-L3, L3-L4, L4-L5, and L5-S1 IVDs, respectively. In this step, a cost-sensitive CNN was used to test for imbalances in the 3-class classification data set [30]. Relevant mathematical theory is provided in Multimedia Appendix 1.

\section{Results}

We focused on images that showed disk bulge, disk herniation, and normal disks. From Table 1, we can see that the probabilities of disk bulge and disk herniation in the L1-L2 and L2-L3 IVDs are low, and disk bulge tended to occur more commonly in the
L3-L4, L4-L5, and L5-S1 IVDs. The L5-S1 IVD is the most common location of disk herniation. This is probably because it bears more weight and pressure than the other locations.

\section{Discussion}

\section{Principal Findings}

Our system is comprised of 4 steps. First, the system automatically located vertebral bodies (from L1 to S1) in sagittal images by using the faster R-CNN, which was trained on 200 manually cropped images. Our fourfold cross-validations showed $100 \%$ accuracy. This high location accuracy shows that the faster R-CNN method can more accurately locate vertebral bodies than many other methods, such as the Gabor filter bank method [31], which is a method based on measurements of disk signal intensity and structure [7]. Second, the disk positions (from L1-L2 to L5-S1) in each axial image were calculated based on the equations for coordinate conversion. We achieved an accuracy of $100 \%$. Third, the system automatically segmented IVD ROIs in axial MR images by using the faster R-CNN, which was trained on 1300 manually boxed images that included all five types of disks (from L1-L2 to L5-S1) and the disk conditions (normal, herniation, and bulge). The mean average precision [21] reached $100 \%$. This high accuracy was the result of the excellent performance of the faster R-CNN. Finally, all ROI images were classified as normal, bulge, and herniation by using ResNet101. The average accuracies for the 3-class classification of the L1-L2, L2-L3, L3-L4, L4-L5, and L5-S1 IVDs were 92.7\%, 84.4\%, 92.1\%, 90.4\%, and 84.2\%, respectively. All relevant results are shown in Figure 4. Previous studies have mainly focused on comparing IVDs affected by 1 disease (disk bulge or herniation) with normal IVDs. This is known as a binary classification. For example, the performance value of one IVD classification system was $86.5 \%$, and this was based on a sparse shape reconstruction from a statistical shape model [32]. Additionally, an accuracy of $92.78 \%$ was reported by a study that classified normal disks and disk bulge by using a program called IVD Descriptor [13]. Compared to the accuracies of these previous studies, our accuracies were roughly the same or slightly inferior. This was mainly because a 3-class classification system is often less accurate than a binary classification system. 
Figure 4. Results of the 3-class classification (normal disk, disk bulge, and disk herniation). (A) The average accuracies of the classification system (calculated using ResNet101) for the following five categories: L1-L2, L2-L3, L3-L4, L4-L5 and L5-S1. The rows and columns of all heat maps represent ground truth labels and predicted labels, respectively. The x-axis shows the five intervertebral disks. (B) A heat map of the classification accuracies for category L1-L2. The color scale expresses the accuracy. (C) A heat map of the classification accuracies for category L2-L3. The color scale expresses the accuracy. (D) A heat map of the classification accuracies for category L3-L4. The color scale expresses the accuracy. (E) A heat map of the classification accuracies for category L4-L5. The color scale expresses the accuracy. (F) A heat map of the classification accuracies for category L5-S1. The color scale expresses the accuracy. L: lumbar vertebra; S: sacral.

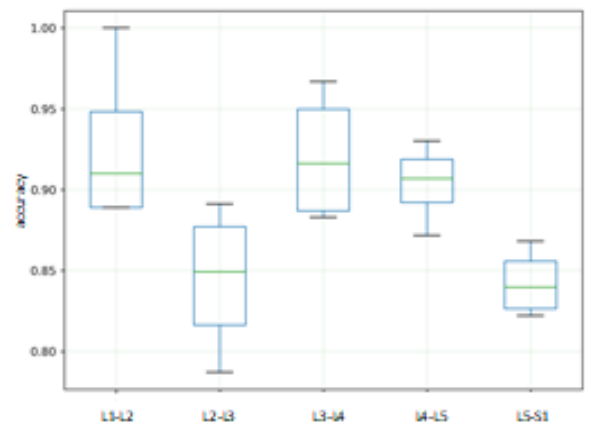

A

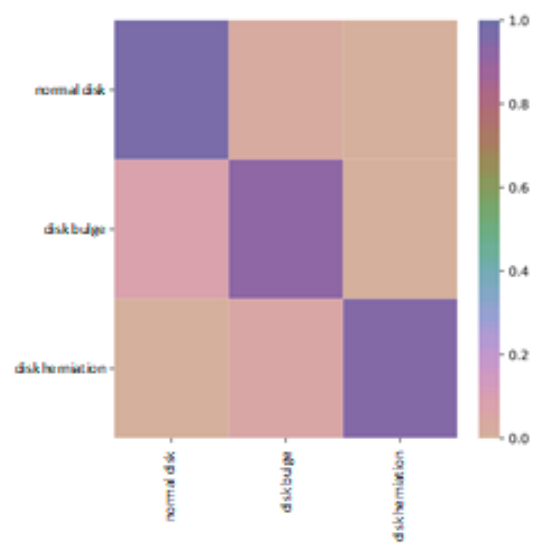

D

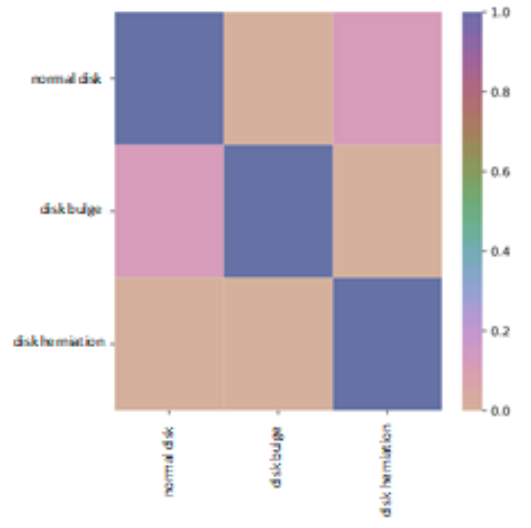

B

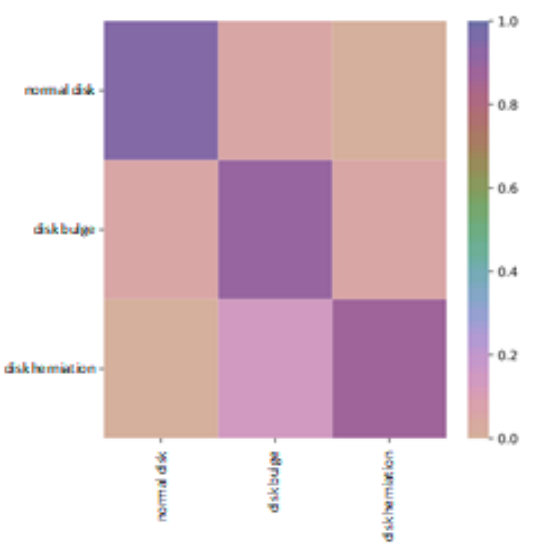

$\mathrm{E}$

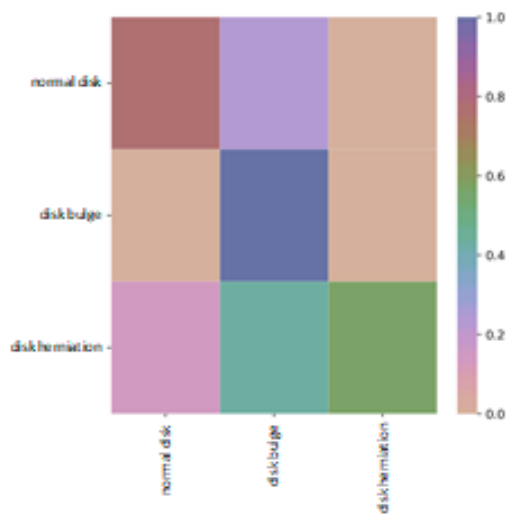

C

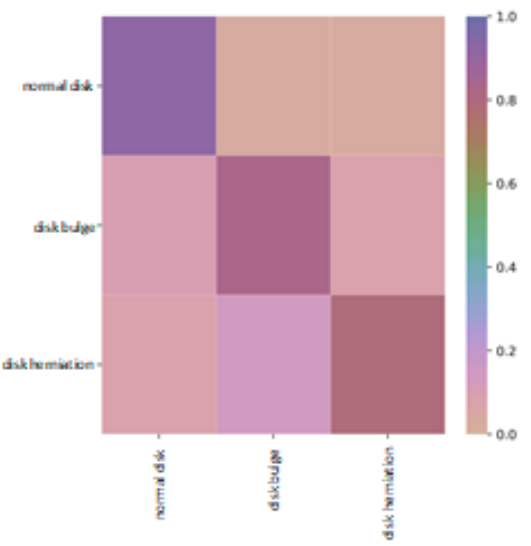

$\mathrm{F}$
Based on our results, the classification accuracies for the L2-L3 and L5-S1 IVDs were lower than those for other disks. The shape of a normal disk is somewhat different from the L1-L2 to L5-S1 IVDs. With regard to the L2-L3 disks, several images were blurry, and it was difficult to identify subtle differences. This, coupled with our small sample of herniated disks, had a considerable impact on our classification accuracy. Data quality may become a crucial factor that could restrict the performance of algorithms used in research [33]. With regard to the L5-S1 disks, the normal disks were similar in shape to that of bulged disks in axial images. There were also a few images that were wrongfully classified by our system, which resulted in a lower classification accuracy.

\section{Web-Based Diagnosis System}

We used the Django framework [34] to develop an automatic diagnosis system for radiologists that could analyze inputted medical images and show results as normalized diagnosis reports (a PDF file). The appearance and functions of the reports are shown in Figure 5. This system can be deployed in multiple radiology departments to analyze patients' lumbar MR images and collect more images to improve radiologists' IVD interpretation performance. This system is freely available [35]. 
Figure 5. Appearance and functions of the reports of the web-based automatic diagnostic system. (A) This is the page for uploading a folder. (B) Diagnostic results in tabular form. (C) The diagnostic report in the Unified format. LDP: lumbar disk protrusion.

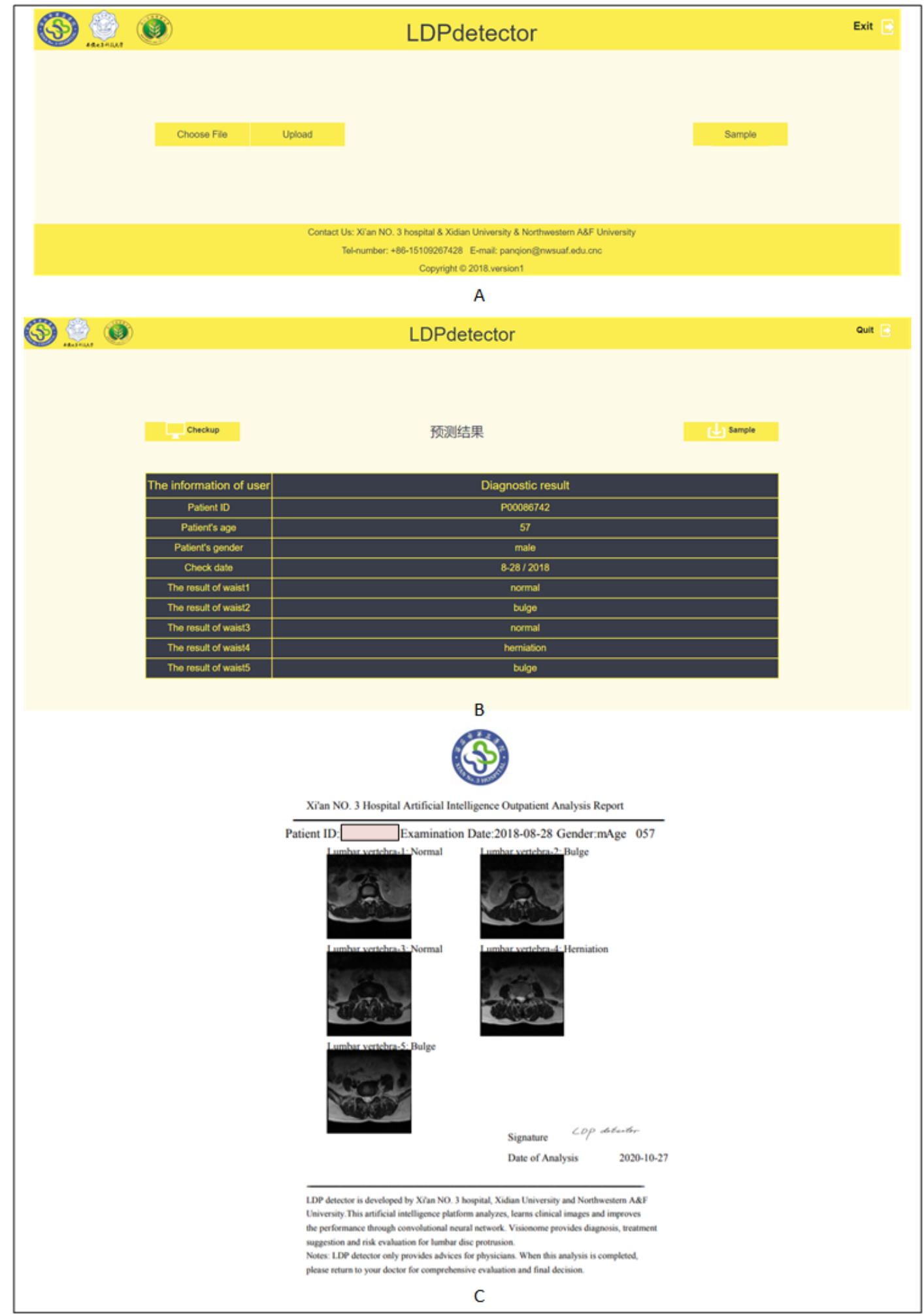

In this paper, we present an automatic diagnosis system for diagnosing disk bulge and disk herniation with axial MR images via deep convolutional neural networks. This system can automatically determine the position and the condition of IVDs in axial MR images. Therefore, this system could help reduce the workloads of radiologists by analyzing lumbar MR images via a standardized method. In addition, this system can be expanded to analyze other types of lumbar diseases, such as cervical spondylosis. However, there are some limitations to using this system. Data from this system could be fundamentally limited by the quality of images (eg, when the image is blurry), making it difficult to identify subtle differences. The system is also limited by the size of the total data set, as it is relatively small for deep convolutional neural networks. Our future work will focus on the following two aspects: (1) developing this system by using a more targeted method that analyzes the 
specific features of MR images, and (2) gathering more MR diagnosis system. images to train a more practical and complete automatic

\section{Acknowledgments}

This study was funded by the National Key Research and Development Program of China (grant 2018YFC0116500).

\section{Authors' Contributions}

YJG designed the study. QP and KZ conducted the study. YW, ZD, and YJG collected and labeled the data. KZ, LH, ZD, QP, and LZ were responsible for coding and analyzing the results. QP and KZ cowrote the manuscript. XHW, KZ and QP critically reviewed and revised the manuscript. All authors discussed the results and commented on the manuscript.

\section{Conflicts of Interest}

None declared.

\section{Multimedia Appendix 1}

Supplementary materials.

[DOCX File, 16 KB-Multimedia Appendix 1]

\section{References}

1. Vos T, Flaxman A, Naghavi M, Lozano R, Michaud C, Ezzati M, et al. Years lived with disability (YLDs) for 1160 sequelae of 289 diseases and injuries 1990-2010: a systematic analysis for the Global Burden of Disease Study 2010. Lancet 2012 Dec 15;380(9859):2163-2196 [FREE Full text] [doi: 10.1016/S0140-6736(12)61729-2] [Medline: 23245607]

2. Yang H, Liu H, Li Z, Zhang K, Wang J, Wang H, et al. Low back pain associated with lumbar disc herniation: role of moderately degenerative disc and annulus fibrous tears. Int J Clin Exp Med 2015 Feb 15;8(2):1634-1644 [FREE Full text] [Medline: 25932092]

3. Li W, Yang Y, Zhang K, Long E, He L, Zhang L, et al. Dense anatomical annotation of slit-lamp images improves the performance of deep learning for the diagnosis of ophthalmic disorders. Nat Biomed Eng 2020 Aug;4(8):767-777. [doi: 10.1038/s41551-020-0577-y] [Medline: 32572198]

4. Wang L, Zhang K, Liu X, Long E, Jiang J, An Y, et al. Comparative analysis of image classification methods for automatic diagnosis of ophthalmic images. Sci Rep 2017 Jan 31;7:41545 [FREE Full text] [doi: 10.1038/srep41545] [Medline: 28139688]

5. Talo M, Baloglu UB, Yıldırım Ö, Rajendra Acharya U. Application of deep transfer learning for automated brain abnormality classification using MR images. Cogn Syst Res 2019 May;54:176-188. [doi: 10.1016/j.cogsys.2018.12.007]

6. Lu J, Pedemonte S, Bizzo B, Doyle S, Andriole K, Michalski M, et al. DeepSPINE: Automated Lumbar Vertebral Segmentation, Disc-level Designation, and Spinal Stenosis Grading Using Deep Learning. 2018 Presented at: Machine Learning for Healthcare Conference; August 17-18, 2018; Palo Alto, California, USA.

7. Koh J, Chaudhary V, Dhillon G. Disc herniation diagnosis in MRI using a CAD framework and a two-level classifier. Int J Comput Assist Radiol Surg 2012 Nov;7(6):861-869. [doi: 10.1007/s11548-012-0674-9] [Medline: 22392057]

8. Alomari RS, Corso JJ, Chaudhary V, Dhillon G. Computer-aided diagnosis of lumbar disc pathology from clinical lower spine MRI. Int J Comput Assist Radiol Surg 2010 May;5(3):287-293. [doi: 10.1007/s11548-009-0396-9] [Medline: 20033498]

9. Hashia B, Mir AH. Texture features' based classification of MR images of normal and herniated intervertebral discs. Multimed Tools Appl 2018 Dec 11;79(21-22):15171-15190. [doi: 10.1007/s11042-018-7011-4]

10. Ruiz-España S, Arana E, Moratal D. Semiautomatic computer-aided classification of degenerative lumbar spine disease in magnetic resonance imaging. Comput Biol Med 2015 Jul;62:196-205. [doi: 10.1016/j.compbiomed.2015.04.028] [Medline: 25957744]

11. Preetha J, Selvarajan S. Computer aided diagnostic system for automatic cervical disc herniation classification. J Med Imaging Health Inform 2016 Nov 01;6(7):1589-1593. [doi: 10.1166/jmihi.2016.1855]

12. Alomari RS, Corso JJ, Chaudhary V, Dhillon G. Automatic diagnosis of lumbar disc herniation with shape and appearance features from MRI. 2010 Mar 09 Presented at: SPIE Medical Imaging 2010: Computer-Aided Diagnosis; February 13-18, 2010; San Diego, California, United States. [doi: 10.1117/12.842199]

13. Beulah A, Sharmila TS, Pramod VK. Disc bulge diagnostic model in axial lumbar MR images using Intervertebral disc Descriptor (IdD). Multimed Tools Appl 2018 Mar 23;77(20):27215-27230. [doi: 10.1007/s11042-018-5914-8]

14. Gulshan V, Peng L, Coram M, Stumpe MC, Wu D, Narayanaswamy A, et al. Development and validation of a deep learning algorithm for detection of diabetic retinopathy in retinal fundus photographs. JAMA 2016 Dec 13;316(22):2402-2410. [doi: 10.1001/jama.2016.17216] [Medline: 27898976] 
15. Zhang K, Liu X, Liu F, He L, Zhang L, Yang Y, et al. An interpretable and expandable deep learning diagnostic system for multiple ocular diseases: Qualitative study. J Med Internet Res 2018 Nov 14;20(11):e11144 [FREE Full text] [doi: 10.2196/11144] [Medline: 30429111]

16. Esteva A, Kuprel B, Novoa RA, Ko J, Swetter SM, Blau HM, et al. Dermatologist-level classification of skin cancer with deep neural networks. Nature 2017 Feb 02;542(7639):115-118. [doi: 10.1038/nature21056] [Medline: 28117445]

17. Abdel-Hamid O, Mohamed A, Jiang H, Deng L, Penn G, Yu D. Convolutional Neural Networks for Speech Recognition. IEEE/ACM Transactions on Audio Speech and Language Process 2014 Oct;22(10):1533-1545. [doi: 10.1109/taslp.2014.2339736]

18. Liu W, Wang Z, Liu X, Zeng N, Liu Y, Alsaadi FE. A survey of deep neural network architectures and their applications. Neurocomputing 2017 Apr;234:11-26. [doi: 10.1016/j.neucom.2016.12.038]

19. Ren S, He K, Girshick R, Sun J. Faster R-CNN: Towards real-time object detection with region proposal networks. IEEE Trans Pattern Anal Mach Intell 2017 Jun;39(6):1137-1149. [doi: 10.1109/TPAMI.2016.2577031] [Medline: 27295650]

20. Zhang K, Li X, He L, Guo C, Yang Y, Dong Z, et al. A human-in-the-loop deep learning paradigm for synergic visual evaluation in children. Neural Netw 2020 Feb;122:163-173. [doi: 10.1016/j.neunet.2019.10.003] [Medline: 31683144]

21. Yang J, Zhang K, Fan H, Huang Z, Xiang Y, Yang J, et al. Development and validation of deep learning algorithms for scoliosis screening using back images. Commun Biol 2019 Oct 25;2:390 [FREE Full text] [doi: 10.1038/s42003-019-0635-8] [Medline: 31667364]

22. Girshick R, Donahue J, Darrell T, Malik J. Rich feature hierarchies for accurate object detection and semantic segmentation. 2014 Presented at: Internaltional Conference on computer vision and pattern recognition; June 24-27, 2014; Columbus, OH, USA p. 580-587. [doi: 10.1109/cvpr.2014.81]

23. Girshick R. Fast R-CNN. 2015 Presented at: IEEE International Conference on Computer Vision; December 13-16, 2015; Santiago, Chile p. 1440-1448. [doi: 10.1109/iccv.2015.169]

24. Jia Y, Shelhamer E, Donahue J, Karayev S, Long J, Girshick R, et al. Caffe: Convolutional Architecture for Fast Feature Embedding. 2014 Nov Presented at: 2014 ACM Multimedia Conference; November 2014; Orlando, Florida, USA p. 675-678. [doi: 10.1145/2647868.2654889]

25. Zhang X, Zhang K, Lin D, Zhu Y, Chen C, He L, et al. Artificial intelligence deciphers codes for color and odor perceptions based on large-scale chemoinformatic data. Gigascience 2020 Feb 01;9(2):giaa011 [FREE Full text] [doi: 10.1093/gigascience/giaa011] [Medline: 32101298]

26. Zhang K, Liu X, Jiang J, Li W, Wang S, Liu L, et al. Prediction of postoperative complications of pediatric cataract patients using data mining. J Transl Med 2019 Jan 03;17(1):2 [FREE Full text] [doi: 10.1186/s12967-018-1758-2] [Medline: 30602368]

27. He K, Zhang X, Ren S, Sun J. Deep Residual Learning for Image Recognition. 2015 Presented at: Internaltional Conference on Computer Vision and Pattern Recognition; June 8-10, 2015; Boston, Massachusetts, USA. [doi: 10.1109/cvpr.2016.90]

28. Bi L, Kim J, Ahn E, Feng D. Automatic skin lesion analysis using large-scale dermoscopy images and deep residual networks. arXiv. Preprint posted online on March 17, 2017. [FREE Full text]

29. Wang X, Peng Y, Lu L, Lu Z, Bagheri M, Summers R. ChestX-ray8: Hospital-scale Chest X-ray Database and Benchmarks on Weakly-Supervised Classification and Localization of Common Thorax Diseases. 2017 Presented at: Internaltional Conference on Computer Vision and Pattern Recognition; July 22-25, 2017; Honolulu, Hawaii, USA p. 3462-3471. [doi: 10.1109/cvpr.2017.369]

30. Jiang J, Liu X, Zhang K, Long E, Wang L, Li W, et al. Automatic diagnosis of imbalanced ophthalmic images using a cost-sensitive deep convolutional neural network. Biomed Eng Online 2017 Nov 21;16(1):132 [FREE Full text] [doi: 10.1186/s12938-017-0420-1] [Medline: 29157240]

31. Zhu X, He X, Wang P, He Q, Gao D, Cheng J, et al. A method of localization and segmentation of intervertebral discs in spine MRI based on Gabor filter bank. Biomed Eng Online 2016 Mar 22;15:32 [FREE Full text] [doi: 10.1186/s12938-016-0146-5] [Medline: 27000749]

32. Neubert A, Fripp J, Engstrom C, Schwarz D, Weber M, Crozier S. Statistical shape model reconstruction with sparse anomalous deformations: Application to intervertebral disc herniation. Comput Med Imaging Graph 2015 Dec;46 Pt 1:11-19. [doi: 10.1016/j.compmedimag.2015.05.002] [Medline: 26060085]

33. Cai L, Zhu Y. The Challenges of Data Quality and Data Quality Assessment in the Big Data Era. Data Sci J 2015 May 22;14:2. [doi: 10.5334/dsj-2015-002]

34. Holovaty A, Kaplan-Moss J. The Definitive Guide to Django: Web Development Done Right. USA: Apress; 2009.

35. He L, Dong Z, Zhang L, Pan Q, Zhang K. Lumbar Disk Protrusion Detection System. URL: http://121.46.19.53/

\section{Abbreviations}

CNN: convolutional neural network

DICOM: Digital Imaging and Communications in Medicine

IVD: intervertebral disk

L: lumbar vertebra 
MR: magnetic resonance

MRI: magnetic resonance imaging

R-CNN: region-based convolutional neural network

ROI: region of interest

S: sacral vertebra

Edited by G Eysenbach, $Q$ Zeng; submitted 18.05.19; peer-reviewed by G Lim, S Kim, Z Li; comments to author 02.09.20; revised version received 27.10.20; accepted 15.04.21; published 21.05.21

Please cite as:

Pan Q, Zhang K, He L, Dong Z, Zhang L, Wu X, Wu Y, Gao Y

Automatically Diagnosing Disk Bulge and Disk Herniation With Lumbar Magnetic Resonance Images by Using Deep Convolutional

Neural Networks: Method Development Study

JMIR Med Inform 2021;9(5):e14755

URL: https://medinform.jmir.org/2021/5/e14755

doi: $10.2196 / 14755$

PMID:

(C)iong Pan, Kai Zhang, Lin He, Zhou Dong, Lei Zhang, Xiaohang Wu, Yi Wu, Yanjun Gao. Originally published in JMIR Medical Informatics (https://medinform.jmir.org), 21.05.2021. This is an open-access article distributed under the terms of the Creative Commons Attribution License (https://creativecommons.org/licenses/by/4.0/), which permits unrestricted use, distribution, and reproduction in any medium, provided the original work, first published in JMIR Medical Informatics, is properly cited. The complete bibliographic information, a link to the original publication on https://medinform.jmir.org/, as well as this copyright and license information must be included. 